\title{
Juvenile idiopathic arthritis-associated uveitis in the era of biological therapy: how the disease changed in more than 20 years of observation in a tertiary referral center in Rome (Italy)
}

\author{
E. Del Giudice $\cdot$ C. Simio $\cdot$ A. Scala $\cdot$ A. Di Coste $\cdot$ G. La Torre $\cdot$ \\ L. Spadea $\cdot$ R. Lubrano $\cdot$ M. Duse $\cdot$ M. P. Paroli
}

Received: 29 March 2021 / Accepted: 22 September 2021 / Published online: 20 October 2021

(C) The Author(s) 2021

\begin{abstract}
Objectives To describe the ophthalmological characteristics in a Juvenile idiopathic arthritis (JIA) cohort and to evaluate how therapeutic advances have changed the course of the uveitis.

Methods Analysis of a retrospective cohort study of consecutive JIA pediatric patients including JIAassociated uveitis (JIA-U) and comparison with a previous study in the same uveitis center assessed before the wide-spread of biological therapy.

Results The total of 49 JIA patients were analyzed, of whom 18 JIA-U, compared with a JIA-U past cohort of 66 patients. Systemic corticosteroids were used significantly less in the current JIA-U group $(p=0.008)$ than in the past one. JIA-U present cohort
\end{abstract}

E. Del Giudice · R. Lubrano

Department of Maternal Infantile and Urological Sciences, Sapienza University of Rome, Polo Pontino, Italy

C. Simio · A. Scala · L. Spadea · M. P. Paroli ( $₫)$ Department of Sense Organs, Eye Clinic, Uveitis Unit, Sapienza University of Rome, V.le del Policlinico 155, 00161 Rome, Italy

e-mail: mariapia.paroli@uniroma1.it

\section{A. Di Coste $\cdot$ M. Duse}

Department of Maternal Infantile and Urological

Sciences, Sapienza University of Rome, Rome, Italy

G. La Torre

Department of Public Health and Infectious Diseases, Sapienza University of Rome, Rome, Italy was on therapy more frequently with conventional synthetic disease-modifying anti-rheumatic drugs (csDMARDs) than the past group ( $p=0.039)$, mostly treated with methotrexate $(93.3 \%)$. Furthermore, a larger use of biologic disease-modifying anti-rheumatic drugs (bDMARDs) was described in the current JIA-U group $(p=0.005)$ also associated with csDMARDs $(p=0.003)$. Adalimumab was used more $(72.7 \%)$ in the present JIA-U cohort compared to a larger treatment with infliximab $(61.5 \%)$ in the past $(p=0.005)$. Higher number of uveitis recurrences was observed in the previous cohort compared to the current one $(p=0.005)$. Fewer complications were described in this study than in the previous: posterior synechiae $(p=0.007)$, cataract $(p<0.001)$, band keratopathy $(p<0.001)$, and elevated intraocular pressure (IOP) $(p=0.047)$.

Conclusion Current therapies reduced the uveitis recurrences and ocular complications including cataract due also to the lower use of corticosteroids. The new close collaboration with the pediatric rheumatologic center in the same University has contributed to the care improvement and decrease of uveitis complications.

Keywords Juvenile idiopathic arthritis - Uveitis · Ocular complications · Visual prognosis $\cdot$ Biological therapy 


\section{Introduction}

JIA is the most common chronic rheumatic disease in children with an incidence of $8.2(7.5-9.0) / 100000$ cases under 16 years of age, and an annual prevalence of approximately 70.2 (16-140)/100000 [1]. Chronic anterior uveitis (CAU) is the most frequent extraarticular manifestation of JIA and its incidence is thought to be approximately $1 / 10000$ with some evidence that it is less frequent in Asian populations affected by JIA $[2,3]$.

The reduction of visual acuity is mainly due to complications resulting from CAU as well as side effects owed to local steroid drugs used to contrast it [4]. The main risk factor for visual impairment is chronic intraocular inflammation, therefore all the therapeutic options are aimed to reduce or to resolve it. Nevertheless, the management strategies of JIArelated uveitis remain a relevant clinical challenge [5].

The first-line standard therapy is topical corticosteroid, even though up to $60 \%$ of the children need further therapies to achieve quiescence [6]. Sometimes systemic corticosteroids are also used for a short time in severe disease, but the next step is usually the immunosuppressive therapy, as disease-modifying anti-rheumatic drugs (DMARDs), mainly using methotrexate (MTX), or other immunosuppressive drugs in MTX-resistant cases [6]. This therapy is usually able to solve the recalcitrant cases achieving a good control of the uveitis in approximately $70-80 \%$ of patients within 3-4 months [7, 8]. However, if the DMARDs are not effective, the biological therapies are considered an appropriate third-line treatment [4]. Currently, TNF- $\alpha$ blocking agents are the main biologics used as treatment of chronic childhood uveitis although in unresponsive JIA uveitis cases other biological drugs options are available [9-12].

The aim of the study was to describe the characteristics of a JIA pediatric population and to assess how therapeutic advances have changed the uveitis course by comparing with a previous cohort evaluated before the wide-spread use of biological therapy in the same uveitis center.

\section{Methods}

Patients and study design

We conducted a retrospective analysis of all consecutive pediatric patients with suspected diagnosis of JIA referred to the Department of Pediatrics and to the Department of Sense Organs, Eye Clinic, Uveitis Unit (Policlinico Umberto I, Sapienza University of Rome), from April 2012 to July 2018. Patients with diagnosis of JIA according to the International League of Associations for Rheumatology criteria were eligible to participate $[12,13]$. The whole cohort was subdivided into two groups: patients with only JIA (group I) and patients with JIA-associated uveitis (JIA-U) (group II). Moreover, data about the JIA-U group were compared to a previous JIA-U cohort (group III) observed from July 1995 to March 2012 in the same referral uveitis center already reported in a published study [14].

Following the close collaboration with the pediatric rheumatologic center in the same University, all JIA patients were assessed by a pediatric rheumatologist and an ophthalmologist at JIA diagnosis and then at every follow-up. All examinations were performed in the same center and by the same group of physicians.

Active uveitis was defined when at least one eye showed an anterior chamber (AC) cells grade above 0 according to the SUN (Standardization of Uveitis Nomenclature) criteria as indicated by the ophthalmologist [15].

Each patient was observed according to the ophthalmological screening uveitis risk for JIA, whereby checks depend on age at disease onset, ILAR reference category, ANA (antinuclear antibodies) positivity, and disease duration $[10,16,17]$.

Demographic data and disease-related variables including sex, JIA disease subtype according to ILAR category [12], age at JIA diagnosis and uveitis diagnosis, JIA and uveitis disease duration, were assessed for each patient.

The JIA uveitis's characteristics and visual acuity were described: date of uveitis diagnosis, anatomical involvement (anterior, intermediate, posterior, panuveitis), laterality, as well as presence of ocular complications and rates of uveitis relapses. Moreover the activity ocular indices were collected for each affected eye according to the SUN International Working Group: the total number of $\mathrm{AC}$ (anterior 
chamber) cells, AC cell grade before and after pupil dilatation (grade $0(<1$ cells), grade $0.5+(1-5$ cells $)$, $1+(6-15$ cells $), 2+(16-25$ cells $), 3+(26-50$ cells $), 4+(>50$ cells $)[15,18]$. Each patient underwent a routine laboratory examination at the first examination and during follow-up. According to clinical history, JIA subtypes and uveitis features, other serological, and immunological exams were analyzed, such as erythrocyte sedimentation rate [ESR], C-reactive Protein [CRP], and ANA [antinuclear antibodies]).

Furthermore, data about the use of conventional synthetic disease-modifying anti-rheumatic drugs (csDMARDs), biologic disease-modifying anti-rheumatic drugs (bDMARDs), or a combination therapy (csDMARD plus bDMARD) were analyzed. Information about the csDMARD used (methotrexate [MTX], azathioprine [AZA], salazopyrine [SSZ]) and bDMARD (adalimumab, infliximab, etanercept, tocilizumab, abatacept), as well as concomitant treatment with mydriatic drops and corticosteroids (topical and/ or systemic), non-steroidal anti-inflammatory drugs (NSAIDs), mean therapy duration and mean time between the introduction of csDMARD and the bDMARD were also recorded.

According to the current guidelines, the first-line treatment approach was the use of topical steroids and mydriatics, then systemic immunosuppressive therapy (mostly methotrexate), and, in case of limited clinical response, the additional use of a biological agent, largely TNF alfa blockers. In case of inadequate response to one biological agent, different possibilities of switch were considered according to the literature $[4,10]$.

All JIA patients underwent ophthalmological examinations following the international recommendations: high-risk children screening every three months (oligoarthritis, polyarthritis rheumatoid factor negative, psoriatic arthritis, or undifferentiated arthritis who are also antinuclear antibody (ANA) positive, younger than 7 years of age at JIA onset, and have JIA duration of 4 years or less); low or moderate risk children screening every 6-12 months (those with high-risk JIA categories but with ANA negative, age of 7 years or older at JIA onset, or have JIA duration of more than 4 years, or systemic JIA or polyarthritis rheumatoid factor positive or enthesitis-related arthritis) [10].
JIA-U patients were then examined at variable interval time depending on therapeutic response and uveitis severity.

The ophthalmological examination included measurement of BCVA (best correct visual acuity) with Snellen's chart at $3 \mathrm{~m}$; examination of the anterior segment with slit-lamp biomicroscopy and intraocular pressure assessment using Goldmann applanation tonometer; fundus examination using bilateral indirect ophthalmoscope. The evaluation of ocular inflammation was based on the cellular activity in the anterior chamber (PK, cells, and flare) according to SUN criteria. Some patients underwent further investigations such as spectral-domain optic coherence tomography (OCT), retinal fluorangiography (FA), and indocyanine angiography (ICGA) when required by the course of uveitis.

\section{Statistical analysis}

All data were summarized and displayed as mean \pm SD for the continuous variables. Categorical data were expressed as frequencies and percentages.

Comparison of groups was performed using the Student $\mathrm{t}$ test for continuous variables normally distributed and Mann-Whitney U test for not normally distributed. The chi-square test was used to evaluate the differences for categorical variables.

An assessment of the correlation between JIA and JIA-U groups and specific variables was performed using the Pearson and Spearman rank correlation. Finally, we performed a stepwise multivariate linear regression analysis that evaluated all variables with a significant bivariate relationship (defined by $p<0.05$ ) with JIA or JIA-U, as well as variables known to influence uveitis, for inclusion in the model. Statistical significance was set at $\mathrm{p}$ value $<0.05$.

The SPSS statistical package version 25 was used to perform all statistical evaluations (SPSS Inc., Chicago, IL).

\section{Ethical considerations}

The study complied with the Declaration of Helsinki and was approved by ethic committee. 


\section{Results}

The baseline characteristics of the present cohort are shown in Table 1 and all study samples including the past JIA-U cohort by Paroli MP in Table 2.

The present cohort included 49 children with a diagnosis of JIA according to the International League of Associations for Rheumatology criteria [12]. Patients affected from JIA associated with uveitis were 18/49 (36.7\%) (On a total of 31 eyes) (group II, JIA-U) while $31 / 49(63.3 \%)$ children had only a diagnosis of JIA (group I).

Overall, the present study cohort was characterized by marked female prevalence in both groups, although mostly in the group II, as well as a higher frequency of oligoarthritis and ANA positive according to ILAR criteria. In all cohort, the mean follow-up duration was $5.1 \pm 3.8$ years. The mean age at the JIA diagnosis was $6.3 \pm 4.3$ years with a JIA disease duration, at last, follow-up of $5.3 \pm 3.7$ years. The mean interval between JIA and uveitis diagnosis was $1.8 \pm 2.1$ years. A lower age at JIA diagnosis was observed in patients with associated uveitis, in particular a mean age at diagnosis of JIA less than 8 years ( $p=0.0001)$. In $15 / 18(83.3 \%)$ patients of the group II, the JIA diagnosis preceded the uveitis, while only in one patient $(5.6 \%)$ the ocular involvement appeared one year before arthritis, and in two patients (11.1\%) uveitis was detected at the same time of initial diagnosis of JIA. JIA was diagnosed mostly between 6 and 10 years of age in $38.7 \%$ of the whole cohort of 49 patients. Laboratory tests such as the mean value of (CRP) and (ESR) at uveitis' diagnosis and at 6 months of follow-up were compared between two groups I and II, JIA and JIA-U, showing no significant differences (Table 1).

During the study period (April 2012-July 2018), a bilateral eye involvement was reported for 13/18 $(72.2 \%)$ patients with a total number of 31 eyes with uveitis. The baseline characteristics of JIA-U patients (group II) and related JIA uveitis features are summarized in Table 3.

Ocular complications were observed more frequently in oligoarticular JIA subtype and the mean age at the time of first ocular complications was $6 \pm 2.7$ years. Moreover, structural ocular complications were observed all at follow-up, in 8/18 (44.4\%) patients and 13 eyes (41.9\% of 31).

The majority of JIA-U patients $(62.5 \%)$ developed only one structural ocular complication, while $12.5 \%$ and $25 \%$ of children, respectively, had two or three of them. The frequencies of ocular complications were $37.5 \%$ at 3 months, $12.5 \%$ at 6 months, and $50 \%$ at $>1$ year from the uveitis diagnosis, with a mean interval time between them of $19.3 \pm 18.3$ months. More than two-thirds of JIA-U patients $(13 / 18,72.2 \%)$ were ANA positive and 6 of whom $(6 / 13,46.2 \%)$

Table 1 Main baseline patients' characteristics of the present cohort: patients with only JIA (group I) and patients with JIAassociated uveitis (JIA-U) (group II)

\begin{tabular}{|c|c|c|c|}
\hline & \multicolumn{2}{|c|}{ All Cohort $(n=49)$} & \multirow[b]{2}{*}{ p value } \\
\hline & $\begin{array}{l}\text { JIA (group I) } \\
(\mathbf{n}=\mathbf{3 1})\end{array}$ & $\begin{array}{l}\text { JIA-U (group II) } \\
(n=18)\end{array}$ & \\
\hline Female sex, n (\%) & $20(64.5 \%)$ & $15(83.3 \%)$ & 0.140 \\
\hline Oligoarthritis, n (\%) & $18(58 \%)$ & $11(61.1 \%)$ & 0.834 \\
\hline Age at JIA diagnosis (mean \pm SD), years & $7.9 \pm 4.3$ & $3.9 \pm 2.4$ & $<0.001$ \\
\hline Age at uveitis diagnosis (mean $\pm \mathrm{SD}$ ), years & - & $4.9 \pm 3.0$ & \\
\hline Time between JIA and uveitis diagnosis (mean \pm SD), years & - & $1.8 \pm 2.1$ & \\
\hline ANA positive, $\mathbf{n}(\%)$ & $16(51.6 \%)$ & $13(72.2 \%)$ & 0.0157 \\
\hline CRP $(\geq 6000 \mu \mathrm{g} / \mathrm{L})$ at the time of uveitis diagnosis, $n(\%)$ & $11(35.5 \%)$ & $7(38.8 \%)$ & 0.539 \\
\hline ESR $(\geq 20 \mathrm{~mm} / \mathrm{h})$ at the time of uveitis diagnosis, $\mathbf{n}(\%)$ & $13(41.9 \%)$ & $10(55.6 \%)$ & 0.253 \\
\hline CRP $(\geq 6000 \mu \mathrm{g} / \mathrm{L})$ at 6 months follow-up from uveitis diagnosis, $n(\%)$ & $19(61.3 \%)$ & $12(66.7 \%)$ & $>0.99$ \\
\hline ESR $(\geq 20 \mathrm{~mm} / \mathrm{h})$ at 6 months follow-up from uveitis diagnosis, $\mathrm{n}(\%)$ & $12(38.7 \%)$ & $10(55.5 \%)$ & 0.41 \\
\hline
\end{tabular}


Table 2 Main baseline patients' characteristics comparing the present JIA-U cohort (group II) to the JIA-U past cohort pre-dating wide-spread use of biologic therapies (group III)*

\begin{tabular}{|c|c|c|c|}
\hline & $\begin{array}{l}\text { JIA-U present cohort } \\
\text { (group II) } \\
(\mathbf{n}=\mathbf{1 8})\end{array}$ & $\begin{array}{l}\text { JIA-U past cohort } \\
\text { (group III) } \\
(\mathbf{n}=69)\end{array}$ & p value \\
\hline Female sex & $15(83.3 \%)$ & $55(79.7 \%)$ & 0.512 \\
\hline Age at JIA diagnosis (mean \pm SD), years & $3.9 \pm 2.4$ & $4.0 \pm 0.8$ & 0.730 \\
\hline Age at uveitis diagnosis (mean \pm SD), years & $4.9 \pm 3.0$ & $3.0 \pm 0.5$ & $<0.001$ \\
\hline $\begin{array}{l}\text { Time interval between JIA and uveitis diagnosis } \\
\text { (mean } \pm \text { SD), years }\end{array}$ & $1.8 \pm 2.1$ & $1.2 \pm 0.25$ & 0.017 \\
\hline Follow-up duration (mean \pm SD), years & $5.1 \pm 3.8$ & $3.17 \pm 4.3$ & 0.086 \\
\hline ANA positive & $13(72.2 \%)$ & $61(88.4 \%)$ & 0.09 \\
\hline Patients on therapy at the last evaluation & $18(100 \%)$ & $69(100 \%)$ & 1.00 \\
\hline Ocular topical therapy & $18(100 \%)$ & $69(100 \%)$ & 1.00 \\
\hline NSAIDs & $13(72.2 \%)$ & $47(68.1 \%)$ & 0.489 \\
\hline Systemic corticosteroids & $6(33.3 \%)$ & $47(68.1 \%)$ & 0.008 \\
\hline csDMARDs monotherapy & $5(27.8 \%)$ & $27(39.1 \%)$ & 0.373 \\
\hline csDMARDS & $15(83.3 \%)$ & $40(58 \%)$ & 0.039 \\
\hline \multicolumn{4}{|l|}{ Type of medications: csDMARDs } \\
\hline MTX & $14(93.3 \%)$ & $23(57.5 \%)$ & 0.035 \\
\hline SSZ & - & - & \\
\hline AZA & $1(6.7 \%)$ & $1(2.5 \%)$ & \\
\hline CsA & - & $5(12.5 \%)$ & \\
\hline $\mathrm{MTX}+\mathrm{CsA}$ & - & $11(27.5 \%)$ & \\
\hline bDMARDs monotherapy & $1(5.6 \%)$ & - & - \\
\hline bDMARD + csDMARD & $10(55.6 \%)$ & $13(18.8 \%)$ & 0.003 \\
\hline \multicolumn{4}{|l|}{ Type of medications: bDMARDs } \\
\hline Adalimumab & $8(72.7 \%)$ & $1(7.7 \%)$ & 0.005 \\
\hline Infliximab & $1(9.1 \%)$ & $8(61.5 \%)$ & \\
\hline Etanercept & - & $3(23.1 \%)$ & \\
\hline Tocilizumab & $1(9.1 \%)$ & $1(7.7 \%)$ & \\
\hline Abatacept & $1(9.1 \%)$ & - & \\
\hline
\end{tabular}

*Paroli MP et al. Ocul Immunol Inflamm. 2015 Feb, p. 23(1):74-81

Except where indicated otherwise, values are the number $(\%)$

Abbreviations: JIA juvenile idiopathic arthritis; ANA antinuclear antibody; NSAIDs non-steroidal anti-inflammatory drugs; MTX methotrexate; SSZ salazopyrine; AZA azathioprine; CsA Cyclosporine; csDMARDS conventional synthetic disease-modifying antirheumatic drugs; bDMARDs biologic disease-modifying anti-rheumatic drugs

developed ocular complications mainly synechiae $(83.3 \%)$.

The visual prognosis of the patients examined appears favorable. In fact, at the end of the follow-up, $83.9 \%$ of eyes reached a vision of $20 / 20$ while low vision of 20/100 was observed in only one eye of a patient with bilateral uveitis, with preservation of a vision of $20 / 20$ in the contralateral eye.
Analyzing the data on visual acuity at the beginning and at the end of the follow-up, it emerges that of the 31 eyes affected by uveitis, 25/31 (80.6\%) eyes had visual acuity between $20 / 20$ and 20/30, 4/31 (12.9\%) eyes between 20/40 and 20/70 and 2/31 (6.5\%) eyes visual acuity reduced to 20/200; while at follow-up $28 / 31(90.3 \%)$ eyes had vision between $20 / 20$ and 20/30, $2 / 31$ (6.4\%) eyes between 20/40 and 20/70 and 
Table 3 JIA-U patients and uveitis characteristics comparing the JIA-U present cohort (group II) to the JIA-U past cohort pre-dating wide-spread use of biologic therapies (group III)*

\begin{tabular}{|c|c|c|c|}
\hline & $\begin{array}{l}\text { JIA-U present cohort } \\
\text { (group II) } \\
(\mathbf{n}=\mathbf{1 8})\end{array}$ & $\begin{array}{l}\text { JIA-U past cohort } \\
\text { (group III) } \\
(\mathbf{n}=69)\end{array}$ & p value \\
\hline Female sex n (\%) & $15(83.3 \%)$ & $55(79.7 \%)$ & 0.512 \\
\hline \multicolumn{4}{|l|}{ JIA subtype and frequency of uveitis, n (\%) } \\
\hline Oligoarthritis & $11(61.1 \%)$ & $44(63.8 \%)$ & \multirow[t]{3}{*}{0.924} \\
\hline Polyarthritis RF + & $3(16.7 \%)$ & $9(13 \%)$ & \\
\hline Polyarthritis RF- & $4(22.2 \%)$ & $16(23.2 \%)$ & \\
\hline \multicolumn{4}{|l|}{ Uveitis involvement, $\mathbf{n}(\%)$} \\
\hline Unilateral & $5(27.8 \%)$ & $22(32 \%)$ & \multirow[t]{2}{*}{0.489} \\
\hline Bilateral & $13(72.2 \%)$ & $47(68 \%)$ & \\
\hline \multicolumn{4}{|l|}{ Uveitis course ${ }^{* *}, \mathbf{n}(\%)$} \\
\hline Acute & $3(16.7 \%)$ & $9(13.0 \%)$ & \multirow[t]{3}{*}{0.924} \\
\hline Recurrent & $1(5.6 \%)$ & $4(5.8 \%)$ & \\
\hline Chronic & $14(77.8 \%)$ & $56(81.2 \%)$ & \\
\hline Affected eyes, n (\%) & $31(86.1 \%)$ & $116(84.1 \%)$ & 0.761 \\
\hline \multicolumn{4}{|l|}{ Ocular complications, $\mathbf{n}(\%)$} \\
\hline Posterior synechiae & $11(35.5 \%)$ & $76(65.5 \%)$ & 0.007 \\
\hline Cataract & $2(6.5 \%)$ & $63(54.3 \%)$ & $<0.001$ \\
\hline Band keratopathy & $3(9.7 \%)$ & $63(54.3 \%)$ & $<0.001$ \\
\hline Elevated IOP & $2(6.5 \%)$ & $25(21.5 \%)$ & 0.047 \\
\hline Macular edema & $4(12.9 \%)$ & $18(15.5 \%)$ & 0.505 \\
\hline Hypotonia & - & $21(18.1 \%)$ & - \\
\hline \multicolumn{4}{|l|}{ Ocular complications during follow-up, $\mathbf{n}(\%)$} \\
\hline Posterior synechiae & $11(35.5 \%)$ & $16(13.8 \%)$ & 0.008 \\
\hline Cataract & $2(6.5 \%)$ & $49(42.2 \%)$ & $<0.001$ \\
\hline Band keratopathy & $3(9.7 \%)$ & $19(16.4 \%)$ & 0.287 \\
\hline Elevated IOP & $2(6.5 \%)$ & $22(19 \%)$ & 0.068 \\
\hline Macular edema & $4(12.9 \%)$ & $16(13.8 \%)$ & 0.558 \\
\hline Hypotonia & - & $16(13.8 \%)$ & - \\
\hline Uveitis recurrences during follow-up (mean \pm SD) & $4.53 \pm 4.47$ & $31.6 \pm 40.7$ & 0.005 \\
\hline
\end{tabular}

*Paroli MP et al. Ocul Immunol Inflamm. 2015 Feb, p. 23(1):74-81

**Uveitis Course (defined by Standardization of uveitis nomenclature 2005): acute an episode characterized by sudden onset and limited duration; recurrent repeated episodes separated by periods of inactivity without treatment $\geq 3$ months in duration; chronic persistent uveitis with relapse within $<3$ months after discontinuing treatment. Jabs DA et al. Am J Ophthalmol 2005, 140(3):509e16

one eye $(3.2 \%)$ visual equal to $20 / 100$. During the follow-up, there was also an improvement in vision in 12 eyes with a mean of $4.5 \pm 2.5$ SD Snellen lines (min 2-max 9) while in 5 a worsening of $5 \pm 2.6 \mathrm{SD}$ Snellen lines (min 2-max 9).
15/18 (83.3\%) JIA-U patients were on therapy with a csDMARD and in $10 / 18(66.7 \%)$ ocular relapses were reported.

In 10/18 (55.6\%) patients a combination therapy with bDMARD was needed, due to lack of uveitis remission. A total of 11 patients were on therapy with bDMARDs. Eight JIA-U patients maintained ocular 
remission on therapy with adalimumab. Only one patient was on monotherapy with bDMARD (adalimumab) due to methotrexate intolerance. In this patient, synechiae were described 5 years before initiation of adalimumab. Furthermore, one patient started therapy with adalimumab and then switched to infliximab to obtain ocular remission. Due to failure or loss of efficacy during anti-TNF $\alpha$ therapy, the use of abatacept or tocilizumab in two children was needed to achieve the uveitis's control. Despite csDMARDsbDMARDs combination therapy, 6/10 (60\%) patients had ocular relapses.

In the present JIA-U cohort on biological medications, we observed a reduction of AC cell grade at follow-up compared to the uveitis diagnosis, specifically $8 / 11(72.7 \%)$ were free of ocular inflammation at 3 months after the bDMARD introduction. BCVA also improved in 7 of 11 patients (63.6\%).

Furthermore, the patients on therapy with csDMARDs plus bDMARDs showed a statistically significant higher frequency of visual acuity improvement than those treated on just csDMARDs $(\mathrm{p}=0.042)$.

Therefore, the data were analyzed to compare JIAU current cohort (group II) with the JIA-U past cohort (group III) referred to patients enrolled in the same uveitis center [14], as shown in Table 2. The past JIA$\mathrm{U}$ cohort of patients presented with lower age at uveitis diagnosis and a shorter time interval between JIA and uveitis diagnosis than the current JIA-U cohort.

All patients in both JIA-U cohorts were on therapy with topical corticosteroid medications (dexamethasone eye drops, 1 to 6 drops/day, depending on severity of uveitis) and mydriatic eye drops (homatropine and/or tropicamide). Almost $70 \%$ of JIA-U past cohort used systemic corticosteroids, significantly more than the current JIA-U group $(p=0.008)$. JIA$\mathrm{U}$ present cohort was on therapy more frequently with csDMARDs than the past group $(p=0.039)$, mostly treated with methotrexate $(93.3 \%)$. Furthermore, a larger use of bDMARDs was described in the current JIA-U group $(\mathrm{p}=0.005)$ also associated with csDMARDs $(p=0.003)$. A larger use of adalimumab (72.7\%) was observed in the present JIA-U cohort compared to a greater use of infliximab $(61.5 \%)$ in the past ( $p=0.005)$ (Table 2).

Moreover, by comparing the JIA-U present cohort (group II) to the cohort pre-dating wide-spread use of biological therapies (group III) by Paroli MP et al. [14]
(Table 3), we found a higher mean number of recurrences during follow-up in the previous cohort than the current one $(31.6 \pm 40.7$ Vs. $4.53 \pm 4.47$, $p=0.005)$. As shown in Table 3 a significantly higher frequency of ocular complications was observed in the past cohort with already evidence in the first ophthalmological examination, whereas no ocular complications were present in the current study at the first observation. Only for the macular edema, no significant difference was described $(p=0.505)$, and hypotonia was not reported in the present our cohort. In the present cohort, the most common ocular complications on the affected eyes were posterior synechiae $(35.5 \%)$, followed by cystoid macular edema (12.9\%), band keratopathy $(9.7 \%)$, and cataract (6.5\%), elevated intraocular pressure (IOP) $(6.5 \%)$.

During the follow-up the posterior synechiae were significantly more frequent in current JIA-U group than in the past one $(\mathrm{p}=0.008)$, compared to a higher frequency of cataract in the past $(<0.001)$.

Overall, we observed a lower number of patients required eye surgery in the JIA-U present group compared to the previous one by Paroli MP et al.

\section{Discussion}

Despite advances in treatment, the JIA-associated uveitis (JIA-U) is still a disabling condition, with possible long-term complications and risk of blindness [19-21].

About one-third of our JIA patients (36.7\%) had associated uveitis; oligoarthritis was the most common form of JIA, affecting $59.2 \%$ of children in all our cohort and $61.1 \%$ of JIA-U, with a definite preference of females (up to $83.3 \%$ in JIA-U) although without statistical difference among JIA children with and without uveitis. A significantly higher frequency of ANA-positive patients was observed in the JIA-U group $(72.2 \%)(p=0.0157)$. Moreover, in $83.3 \%$ of our cases, the diagnosis of JIA preceded the ocular involvement, and we observed a significant correlation between uveitis onset and younger age at JIA diagnosis [13, 22, 23, 23, 24, 24, 25]. Calandra et. Al [25] reported a higher uveitis risk related to younger age at JIA onset, ANA positivity but not to the gender and Saurenmann RK et al. [26] described the patient's age at the time of arthritis onset and ANA positivity in girls but not in boys as factors related to uveitis [27]). 
In our data the mean age of JIA diagnosis in children who developed uveitis (3.9 years) and the mean interval time between JIA and uveitis diagnosis ( \pm 20 months) were in accordance with several studies by Heiligenhaus et al. [17], Papadopoulou M. [28], Taha R. [29] and Cecchin V. [30] (specifically they reported the following data: 3.8 years/ 21 months, 3.4 years/17 months, 3.3 years/18 months, 4.4 years / 1.6 months, respectively).

The age of first uveitis manifestation was also analyzed compared to different age groups, and the mean age at uveitis diagnosis at 5 years of age according to the study by Castagna I et al. [31]. Although, Castagna I et al. evidenced two different peaks of age for the ocular involvement, the first occurred between 4 and 6 years old and the second between 10 and 12 years old. Also, Hoeve Met al. [32] described a second peak around puberty.

Our study is a continuation of the study by Paroli MP. et al. [14] referred to patients enrolled in previous seven years in the same uveitis center in Policlinico Umberto I Sapienza.

Nevertheless, the JIA cohort in the first study was larger, but the mean follow-up was shorter than our new study $(3.17 \pm 4.3$ years Vs. $5.1 \pm 3.8$ years $)$.

A higher frequency of oligoarthritis and a female prevalence in JIA-U patients were confirmed in both studies. A longer time interval between the JIA and uveitis diagnosis was described in the present cohort $(p=0.017)$, maybe due to an early JIA diagnosis and treatment with a delay on ocular inflammation complications.

Regarding the laboratory exams, similarly to the previous study, a higher frequency of ANA positive in JIA-U patients was found: $72.2 \%$ versus $88.4 \%$ of the previous study, while the inflammation index ESR was positive at the uveitis diagnosis in $55.6 \%$ of JIA-U patients in this study compared to $76.5 \%$ of the previous one. In the study by Paroli MP et al., 30\% of affected eyes had at least one ocular complication at first visit, while in this study no patients presented ocular complications at first ophthalmological evaluation and the majority (50\%) developed them at $>1$ year from the uveitis diagnosis, with a mean interval time between them of $19.3 \pm 18.3$ months.

In this study the frequency of ocular complications observed was lower compared to the previous, respectively: posterior synechiae $(35.5 \%$ and $65.5 \%)$, cataract $(6.5 \%$ and $54.3 \%)$, band keratopathy
(9.7\% and 54.3\%), elevated IOP (6.5\% and $21.5 \%)$, and the macular edema (12.9\% and $15.5 \%)$.

The hypotonia, considered as one of the most frequent cause of low vision and blindness in the past, was not described in our study.

The data about the late and lower development of ocular complications such as posterior synechiae, cataract, and band keratopathy could be due to an early diagnosis and the ophthalmological screening for JIA in the last decade.

The limitations of the present study could be the relatively small population and the retrospective design. Nevertheless, the main strength of the study is the comparison between a present JIA-U cohort with a previous JIA-U group of patients enrolled before the wide-spread use of biological therapy in the same uveitis center.

In the past cohort by Paroli MP et al. [14] many patients came from rheumatological centers from different parts of Italy or directly from ophthalmologists with an inevitable delay in JIA diagnosis, while the close collaboration with the pediatric rheumatologic center in the same University has contributed to the care improvement and decrease of ocular complications. Moreover, the reduction and prevalence of the uveitis severity in JIA patients could be related to greater knowledge about JIA uveitis, an early treatment and the introduction of new therapies including biological agents. In fact, the treatment with systemic corticosteroid therapy was significantly decreased in our current cohort compared to the past one $(p=0.008)$ for the use of csDMARDs and bDMARDs, being another possible cause of reductions of cataracts in the most recent cohort.

The importance of early administration of biologic therapy and its role to improve also extraocular manifestations, it has been highlighted also in other forms of pediatric uveitis such as in Behçet's disease, typically managed by topical corticosteroids and mydriatic agents $[33,34]$.

Furthermore, in our present JIA-U cohort on biological therapy, a higher visual acuity improvement was observed, according to data reported to other authors also in other pediatric uveitis of diverse etiologies [35, 36]

The likely different response rates to biological therapy between non-infectious pediatric anterior uveitis of different aetiologies [36], as well as treated with anti- TNF- $\alpha$ agents, infliximab or adalimumab, 
have been described in the literature [37, 38]. Additional comparison studies are needed to clarify all possible differences.

\section{Conclusion}

Although new drugs improved significantly the course of JIA and visual prognosis of associated uveitis, the disease remains a challenge for pediatric rheumatologists and ophthalmologists, and patients are still developing sight-threatening ocular complications. A routine ophthalmological follow-up is therefore required at regular intervals, in order to identify early ocular involvement.

Further studies are needed to better characterize a target therapeutic approach. The appropriate treatment is necessary to reduce the long-term disability and improve the quality of life in JIA-U.

Optimal care of children with JIA and uveitis requires careful and close collaboration between ophthalmologists and rheumatologists to choose the most appropriate systemic therapy. New discoveries on the pathogenesis of JIA-U and the identification of predictive biomarkers could help find a targeted therapy tailored to individual patients and based on their predicted response or risk of disease.

Funding Open access funding provided by Università degli Studi di Roma La Sapienza within the CRUI-CARE Agreement. No specific funding was received from any bodies in the public, commercial, or not-for-profit sectors to carry out the work described in this manuscript.

Open Access This article is licensed under a Creative Commons Attribution 4.0 International License, which permits use, sharing, adaptation, distribution and reproduction in any medium or format, as long as you give appropriate credit to the original author(s) and the source, provide a link to the Creative Commons licence, and indicate if changes were made. The images or other third party material in this article are included in the article's Creative Commons licence, unless indicated otherwise in a credit line to the material. If material is not included in the article's Creative Commons licence and your intended use is not permitted by statutory regulation or exceeds the permitted use, you will need to obtain permission directly from the copyright holder. To view a copy of this licence, visit http://creativecommons.org/licenses/by/4.0/.

\section{References}

1. Prakken B, Albani S, Martini A (2011) Juvenile idiopathic arthritis. Lancet 377(9783):2138-2149. https://doi.org/10. 1016/s0140-6736(11)60244-4

2. Clarke SLN, Sen ES, Ramanan AV (2016) Juvenile idiopathic arthritis-associated uveitis. Pediatr Rheumatol Online J 14(1):27. https://doi.org/10.1186/s12969-0160088-2

3. Del Giudice E, Swart JF, Wulffraat NM (2017) Juvenile idiopathic arthritis. Comorbidity Rheum Dis 5:265-288

4. Amin RM, Miserocchi E, Thorne JE et al (2016) Treatment options for juvenile idiopathic arthritis (JIA) associated uveitis. Ocul Immunol Inflamm 24(1):81-90. https://doi. org/10.3109/09273948.2015.1077976

5. Constantin T, Foeldvari I, Anton J et al (2018) Consensusbased recommendations for the management of uveitis associated with juvenile idiopathic arthritis: the SHARE initiative. Ann Rheum Dis 77(8):1107-1117. https://doi. org/10.1136/annrheumdis-2018-213131

6. Yu EN, Meniconi ME, Tufail F et al (2005) Outcomes of treatment with immunomodulatory therapy in patients with corticosteroid-resistant juvenile idiopathic arthritis-associated chronic iridocyclitis. Ocul Immunol Inflamm 13(5):353-360. https://doi.org/10.1080/ 09273940590951061

7. Ayuso VK, Van De Winkel EL et al (2011) Relapse rate of uveitis post-methotrexate treatment in juvenile idiopathic arthritis. Am J Ophthalmol 151(2):217-222. https://doi.org/ 10.1016/j.ajo.2010.08.021

8. Heiligenhaus A, Mingels A, Heinz C et al (2007) Methotrexate for uveitis associated with juvenile idiopathic arthritis: value and requirement for additional anti-inflammatory medication. Am J Ophthalmol 17(2):743-748. https://doi.org/10.1177/112067210701700509

9. Smith JA, Thompson DJS, Whitcup SM et al (2005) A randomized, placebo-controlled, double-masked clinical trial of etanercept for the treatment of uveitis associated with juvenile idiopathic arthritis. Arthritis Rheum 53(1):18-23. https://doi.org/10.1002/art.20904

10. Tynjala $P$, Lindahl $P$, Honkanen V et al (2006) Infliximab and etanercept in the treatment of chronic uveitis associated with refractory juvenile idiopathic arthritis. Ann Rheum Dis 66(4):548-550. https://doi.org/10.1136/ard.2006.058248

11. Angeles-Han ST, Ringold S, Beukelman T et al (2019) 2019 American college of rheumatology/arthritis foundation guideline for the screening, monitoring, and treatment of juvenile idiopathic arthritis-associated uveitis. Arthritis Care Res (Hoboken) 71(6):703-716. https://doi.org/10. 1002/acr.23871

12. Ramanan A, Dick AD, Jones AP et al (2017) Adalimumab plus methotrexate for uveitis in juvenile idiopathic arthritis. N Engl J Med 376(17):1637-1646. https://doi.org/10.1056/ nejmoa1614160

13. Petty RE, Southwood TR, Manners P et al (2004) International league of associations for rheumatology classification of juvenile idiopathic arthritis: second revision, Edmonton, 2001. J Rheumatol 31(2):390-392 
14. Ravelli A, Martini A (2007) Juvenile idiopathic arthritis. Lancet 369(9563):767-778. https://doi.org/10.1016/s01406736(07)60363-8

15. Paroli MP, Abbouda A, Restivo L et al (2015) Juvenile idiopathic arthritis-associated uveitis at an Italian tertiary referral center: clinical features and complications. Ocul Immunol Inflamm 23(1):74-81. https://doi.org/10.3109/ 09273948.2013.855798

16. Jabs DA, Nussenblatt RB, Rosenbaum JT (2005) Standardization of uveitis nomenclature (SUN) working group. Standardization of uveitis nomenclature for reporting clinical data. Results of the first international workshop. Am J Ophthalmol 140(3):509e16. https://doi.org/10.1016/j.ajo. 2005.03.057

17. Cassidy J, Kivlin J, Lindsley C, Nocton J (2006) Ophthalmologic examinations in children with juvenile rheumatoid arthritis. Pediatrics 117:1843-1845. https://doi.org/10. 1542/peds.2006-0421

18. Heiligenhaus A, Niewerth M, Ganser G et al (2007) German uveitis in childhood study group. Prevalence and complications of uveitis in juvenile idiopathic arthritis in a population-based nation-wide study in Germany: suggested modification of the current screening guidelines. Rheumatology 46(6):1015-1019. https://doi.org/10.1093/ rheumatology/kem053

19. Deschenes J, Murray PI, Rao NA, Nussenblatt RB (2008) International uveitis study group (IUSG): clinical classification of uveitis. Ocul Immunol Inflamm 16(1):1e2. https:// doi.org/10.1080/09273940801899822

20. Gregory AC 2nd, Kempen JH, Daniel E et al (2013) Systemic immunosuppressive therapy for eye diseases cohort study research group. Ophtalmology 120(1):186-192. https://doi.org/10.1016/j.ophtha.2012.07.052

21. Foeldvari I, Klotsche J, Simonini G et al (2019) Proposal for a definition for response to treatment, inactive disease and damage for JIA associated uveitis based on the validation of a uveitis related JIA outcome measures from the Multinational Interdisciplinary Working Group for uveitis in childhood (MIWGUC). Pediatric Rheumatol Online 17(1):66. https://doi.org/10.1186/s12969-019-0345-2

22. Thorne JE, Woreta F, Kedhar SR et al (2007) Juvenile idiopathic arthritis associated uveitis: incidence of ocular complications and visual acuity loss. Am J Opthalmol 143(5):840-846. https://doi.org/10.1016/j.ajo.2007.01.033

23. Moradi A, Amin RM, Thorne JE (2014) The role of gender in juvenile idiopathic arthritis- associated uveitis. J Ophthalmol. https://doi.org/10.1155/2014/461078

24. Conti G, Chirico V, Porcaro F et al (2019) Frequency and identification of risk factors of uveitis in juvenile idiopathic arthritis: a long-term follow-up study in a cohort of Italian children. J Clin Rheumatol. https://doi.org/10.1097/rhu. 0000000000001104

25. Heiligenhaus A, Heinz C, Edelsten C et al (2013) (2013) Review for disease of the year: epidemiology of juvenile idiopathic arthritis and its associated uveitis: the probable risk factors. Ocul Immunol Inflamm 21(3):180-191. https:// doi.org/10.3109/09273948.2013.791701

26. Calandra S, Gallo MC, Consolaro A et al (2014) Female sex and oligoarthritis category are not risk factors for uveitis in Italian children with juvenile idiopathic arthritis.
J Rheumatol 41(7):1416-1425. https://doi.org/10.3899/ jrheum.131494

27. Saurenmann RK, Levin AV, Feldman BM et al (2010) Risk factors for development of uveitis differ between girls and boys with juvenile idiopathic arthritis. Rheum 62(6):1824-1828. https://doi.org/10.1002/art.27416

28. Angeles-Han ST, Pelajo CF, Vogler LB et al (2013) Risk markers of juvenile idiopathic arthritis-associated uveitis in the childhood arthritis and rheumatology research alliance (CARRA) registry. J Rheumatol 40(12):2088-2096. https:// doi.org/10.3899/jrheum.130302

29. Papadopoulou M, Zetterberg M, Oskarsdottir S et al (2017) Assessment of the outcome of ophthalmological screening for uveitis in a cohort of Swedish children with juvenile idiopathic arthritis. Acta Ophthalmol 95(7):741-747. https://doi.org/10.1111/aos.13388

30. Taha R, Papadopoulou M, Zetterberg M et al (2019) Visual function and quality of life in a cohort of swedish children with juvenile idiopathic arthritis. Clin Ophthalmol 13:2081-2091. https://doi.org/10.2147/opth.s202486

31. Cecchin V, Zannin ME, Ferrari D et al (2018) Longterm safety and efficacy of adalimumab and infliximab for uveitis associated with juvenile idiopathic arthritis. J Rheumatol 45(8):1167-1172. https://doi.org/10.3899/jrheum.171006

32. Castagna I, Roszkowska AM, Alessandrello F et al (2019) Juvenile idiopathic arthritis-associated uveitis: a retrospective analysis from a centre of South Italy. Int. Opthalmol. 40(2):335-342. https://doi.org/10.1007/s10792-019-011848

33. Hoeve M, Kalinina Ayuso V, Schalij-Delfos NE et al (2012) The clinical course of juvenile idiopathic arthritis-associated uveitis in childhood and puberty. Br J Ophthalmol 96(6):852-856. https://doi.org/10.1136/bjophthalmol2011-301023

34. Hiyama T, Harada Y, Doi T, Kiuchi Y (2019) Early administration of adalimumab for paediatric uveitis due to Behçet's disease. Pediatr Rheumatol Online J 17(1):29. https://doi.org/10.1186/s12969-019-0333-6

35. Neri P, Eandi C, Arapi I et al (2013) Long-term control of non-infectious paediatric panuveitis refractory to traditional immunesuppressive therapy successfully treated with Adalimumab (HumiraTM). Clin Exp Rheumatol 31:458-462

36. Lerman MA, Rabinovich CE (2015) The future is now: biologics for non-infectious pediatric anterior uveitis. Pediatr Drugs 17(4):283-301. https://doi.org/10.1007/ s40272-015-0128-2

37. Deitch I, Amer R, Tomkins-Netzer O et al (2018) The effect of anti-tumor necrosis factor alpha agents on the outcome in pediatric uveitis of diverse etiologies. Graefes Arch Clin Exp Ophthalmol 256:801-808. https://doi.org/10.1007/ s00417-018-3928-6

38. Ashkenazy N, Saboo US, Abraham A et al (2019) Successful treatment with infliximab after adalimumab failure in pediatric noninfectious uveitis. J AAPOS 23(3):151.e1151.e5. https://doi.org/10.1016/j.jaapos.2019.02.006

Publisher's Note Springer Nature remains neutral with regard to jurisdictional claims in published maps and institutional affiliations. 